\title{
A symplectic non-squeezing theorem for BBM equation
}

\author{
David Roumégoux
}

Communicated by Yvan Martel, received July 8, 2010.

ABstract. We study the initial value problem for the BBM equation:

$$
\left\{\begin{array}{l}
u_{t}+u_{x}+u u_{x}-u_{t x x}=0 \quad x \in \mathbb{T}, t \in \mathbb{R} \\
u(0, x)=u_{0}(x)
\end{array}\right.
$$

We prove that the BBM equation is globaly well-posed on $H^{s}(\mathbb{T})$ for $s \geq 0$ and a symplectic non-squeezing theorem on $H^{1 / 2}(\mathbb{T})$. That is to say the flow-map $u_{0} \mapsto u(t)$ that associates to initial data $u_{0} \in H^{1 / 2}(\mathbb{T})$ the solution $u$ cannot send a ball into a symplectic cylinder of smaller width.

\section{Contents}

1. Introduction 289

2. Symplectic capacities in Hilbert spaces and non-squeezing theorem 290

3. Application to the BBM equation 298

$\begin{array}{ll}\text { References } & 305\end{array}$

\section{Introduction}

In 1877 Joseph Boussinesq proposed a variety of models for describing the propagation of waves on shallow water surfaces, including what is now refered to as the Korteweg-de Vries (KdV) equation. A scaled KdV equation reads

$$
u_{t}+u_{x}+\varepsilon\left(u u_{x}+u_{x x x}\right)=0 .
$$

The Benjamin-Bona-Mahony (BBM) equation was introduced in [1] as an alternative of the $\mathrm{KdV}$ equation. The main argument to derive the BBM equation is that, to the first order in $\varepsilon$, the scaled $\mathrm{KdV}$ equation is equivalent to

$$
u_{t}+u_{x}+\varepsilon\left(u u_{x}-u_{t x x}\right)=0 .
$$

Indeed, formally we have $u_{t}+u_{x}=O(\varepsilon)$, hence $u_{x x x}=-u_{t x x}+O(\varepsilon)$.

2000 Mathematics Subject Classification. Primary 35; Secondary 47.

Key words and phrases. BBM equation, well-posedness, symplectic non-squeezing theorem.

(c)2010 International Press 
In this article we shall consider the rescaled BBM equation:

$$
u_{t}+u_{x}+u u_{x}-u_{t x x}=0 .
$$

In 2009, Jerry Bona and Nikolay Tzvetkov proved in [2] that BBM equation is globaly well-posed in $H^{s}(\mathbb{R})$ if $s \geq 0$, and not even locally well-posed for negative values of $s$ (see also $[\mathbf{8}]$ ). The result extends to the periodic case (see section 3 below). Let us denote $\Phi_{t}$ the flow map of BBM equation on the circle $\mathbb{T}$. In this article we prove a symplectic non-squeeezing theorem for $\Phi_{t}$. That is, the flow map cannot squeeze a ball of radius $r$ of $H^{1 / 2}(\mathbb{T})$ into a symplectic cylinder of radius $r^{\prime}<r$. Precisely, let $H_{0}^{1 / 2}(\mathbb{T})=\left\{u \in H^{1 / 2} / \int_{\mathbb{T}} u=0\right\}$ with the Hilbert basis

$$
\varphi_{n}^{+}(x)=\sqrt{\frac{n}{\pi\left(n^{2}+1\right)}} \cos (n x), \quad \varphi_{n}^{-}(x)=\sqrt{\frac{n}{\pi\left(n^{2}+1\right)}} \sin (n x) .
$$

Set

$$
\begin{gathered}
B_{r}=\left\{u \in H_{0}^{1 / 2}(\mathbb{T}) /\|u\|_{H^{1 / 2}}<r\right\}, \\
\mathcal{C}_{r, n_{0}}=\left\{u=\sum p_{n} \varphi_{n}^{+}+q_{n} \varphi_{n}^{-} \in H_{0}^{1 / 2}(\mathbb{T}) / p_{n_{0}}^{2}+q_{n_{0}}^{2}<r^{2}\right\} .
\end{gathered}
$$

The goal of this paper is to prove

TheOREM 1.1. If $\Phi_{t}\left(B_{r}\right) \subset \mathcal{C}_{R, n_{0}}$ then $r \leq R$.

S. Kuksin initiated the investigation of non-squeezing results for infinite dimentional Hamiltonian systems (see [7]). In particular he proved that nonlinear wave equation has the non-squeezing property for some nonlinearities. This result were extended to certain stronger nonlinearities by Bourgain [3], and he also proved with a different method that the cubic NLS equation on the circle $\mathbb{T}$ has the nonsqueezing property. Using similar ideas Colliander, Keel, Staffilani, Takaoka and Tao obtained the same result for $\mathrm{KdV}$ equation on $\mathbb{T}$ (see [4]).

In this article we will use the original theorem of Kuksin. In section 2, we present the construction of a capacity on Hilbert spaces introduced by Kuksin in [7]. This capacity is invariant with respect to the flow of some hamiltonian PDEs provided it has the form "linear evolution + compact". As a corollary of this result we get a non-squeezing theorem for these PDEs. Then we apply this theorem to the $\mathrm{BBM}$ equation in section 3. We prove the global wellposedness of BBM equation on $H^{s}(\mathbb{T})$ for $s \geq 0$, and some estimates on the solutions.

\section{Symplectic capacities in Hilbert spaces and non-squeezing theorem}

2.1. The frame work and an abstract non-squeezing theorem. Let $(Z,\langle\cdot, \cdot\rangle)$ be a real Hilbert space with $\left\{\varphi_{j}^{ \pm} / j \geq 1\right\}$ a Hilbert basis. For $n \in \mathbb{N}$ we denote $Z^{n}=\operatorname{Span}\left(\left\{\varphi_{j}^{ \pm} / 1 \leq j \leq n\right\}\right)$, and $\Pi^{n}: Z \rightarrow Z^{n}$ the corresponding projector. We also denote $Z_{n}$ the space such that $Z=Z^{n} \oplus Z_{n}$. Then, every $z \in Z$ admits the unique decomposition $z=z^{n}+z_{n}$ with $z_{n} \in Z_{n}$ and $z^{n} \in Z^{n}$.

We define $J: Z \rightarrow Z$ the skewsymmetric linear operator by

$$
J \varphi_{j}^{ \pm}=\mp \varphi_{j}^{\mp}
$$

and we supply $Z$ with a symplectic structure with the 2 -form $\omega$ defined by $\omega(\xi, \eta)=$ $\langle J \xi, \eta\rangle$.

We take a self-adjoint operator $A$, such that

$$
\forall j \in \mathbb{Z}, A \varphi_{j}^{ \pm}=\lambda_{j} \varphi_{j}^{ \pm}
$$


Define the Hamiltonian

$$
f(z)=\frac{1}{2}\langle A z, z\rangle+h(z)
$$

where $h$ is a smooth function defined on $Z \times \mathbb{R}$. The corresponding Hamiltonian equation has the form

$$
\left\{\begin{array}{l}
\dot{z}=J A z+J \nabla h(z) \\
z(0, \cdot)=z_{0} \in Z
\end{array}\right.
$$

If $Z_{-}$is a Hilbert space, we denote

$$
Z<Z_{-}
$$

if $Z$ is compactly embedded in $Z_{-}$and $\left\{\varphi_{j}^{ \pm}\right\}$is an orthogonal basis of $Z_{-}$(not an orthonormal one!). Clearly $Z$ is dense in $Z_{-}$. We identify $Z$ and its dual $Z^{*}$. Then $\left(Z_{-}\right)^{*}$ can be identified with a subspace $Z_{+}$of $Z$ and we have

$$
Z_{+}<Z<Z_{-} \text {. }
$$

Denote $\|\cdot\|_{-}\left(\right.$resp. $\left.\|\cdot\|_{+}\right)$the norm of $Z_{-}\left(\right.$resp. $\left.Z_{+}\right)$.

We also denote $B_{R}(Z)$ the ball centered at the origin of radius $R$.

We impose the following assumptions:

(H1): The equation (2) defines a $C^{1}$-smooth global flow map $\Phi$ on $Z$. That is, for all $z_{0} \in Z$ the equation (2) has a unique solution $z(t)=\Phi_{t}\left(z_{0}\right)$ for $t \geq 0$, and the flow map $\Phi_{t}: z_{0} \mapsto z(t)$ is $C^{1}$-smooth.

(H2): The flow map $\Phi$ is uniformely bounded. That is for each $R>0$ and $T>0$, there exists $R^{\prime}=R_{R, T}^{\prime}$ such that

$$
\Phi_{t}\left(B_{R}(Z)\right) \subset B_{R^{\prime}}(Z), \quad \text { for }|t| \leq T .
$$

(H3): Writing the flow map $\Phi_{t}=e^{t J A}\left(I+\widetilde{\Phi}_{t}\right)$, we also impose the following compactness assumption : fix $R>0$ and $T>0$, there exists $C_{R, T}$ such that

$$
\forall u_{0}, u_{0}^{\prime} \in B_{R}(Z),\left\|\widetilde{\Phi}_{T}\left(u_{0}\right)-\widetilde{\Phi}_{T}\left(u_{0}^{\prime}\right)\right\|_{Z_{+}} \leq C_{R, T}\left\|u_{0}-u_{0}^{\prime}\right\|_{Z} \cdot
$$

Under these assumptions, it is well known that the flow maps $\Phi_{t}$ preserve the symplectic form.

The aim of this section is to show the following non-squeezing theorem

THEOREM 2.1. Assume $\Phi_{T}$ is the flow map of an equation of the form (2) and satisfies the previous assumptions. If $\Phi_{T}$ sends a ball

$$
B_{r}=\{z \in Z /\|z-\bar{z}\|<r\}, \quad \bar{z} \text { fixed }
$$

into a cylinder

$$
\begin{aligned}
& \mathcal{C}_{R, j_{0}}=\left\{z=\sum p_{j} \varphi_{j}^{+}+q_{j} \varphi_{j}^{-} /\left(p_{j_{0}}-\bar{p}_{j_{0}}\right)^{2}+\left(q_{j_{0}}-\bar{q}_{j_{0}}\right)^{2}<R^{2}\right\} \\
& j_{0}, \bar{p}_{j_{0}}, \bar{q}_{j_{0}} \text { fixed }
\end{aligned}
$$

then $r \leq R$.

In fact, this theorem is a simple version of the conservation of a symplectic capacity on $Z$ by the flow map $\Phi_{T}$ (see subsection 2.3 .2 below) 
REMARK 2.2. This theoreme implies the following fact. Fiw $\varepsilon>0$, a time $T>0$, a Fourier mode $n_{0}$ and $r>0$ (no smallness conditions are imposed on $r$ or $T)$, then there exists $u_{0} \in H^{1 / 2}(\mathbb{T})$ such that

$$
\left\|u_{0}\right\|_{H^{1 / 2}}<r
$$

and

where $u$ solves (2).

$$
\left|\widehat{u(T)}\left(n_{0}\right)\right|>\frac{r-\varepsilon}{\left(n_{0}^{2}+1\right)^{1 / 4}}
$$

The non-squeezing theorem remains true if we don't suppose that the flow map is global in (H1), but the conclusion would be :

either

or

$$
\widehat{\mid u(T)}\left(n_{0}\right) \mid>\frac{r-\varepsilon}{\left(n_{0}^{2}+1\right)^{1 / 4}}
$$

$$
\sup _{0 \leq t \leq T}\|u(t)\|_{H^{1 / 2}}=+\infty \text {. }
$$

So we impose the global wellposedness in time for (2) in order to rule out the second case.

2.2. An approximation lemma. In order to define a capacity, we will need to approximate the flow by finite-dimentional maps. We shall use the following lemma

LEMMA 2.3. Let $\Phi$ the flow at time $T$ of an equation (2) satisfying the previous assumptions. For each $\varepsilon>0$ and $R>0$, there exists $N \in \mathbb{N}$ such that for $u \in B_{R}$ :

$$
\Phi(u)=e^{t J A}\left(I+\widetilde{\Phi}_{\varepsilon}\right)\left(I+\widetilde{\Phi}_{N}\right)(u)
$$

where $\left(I+\widetilde{\Phi}_{\varepsilon}\right)$ and $\left(I+\widetilde{\Phi}_{N}\right)$ are symplectic diffeomorphisms satisfying

$$
\left\|\widetilde{\Phi}_{\varepsilon}(u)\right\| \leq \varepsilon \quad \text { for } u \in\left(I+\widetilde{\Phi}_{N}\right)\left(B_{R}\right)
$$

$$
\left(I+\widetilde{\Phi}_{N}\right)\left(u^{N}+u_{N}\right)=\left(I+\widetilde{\Phi}_{N}\right)\left(u^{N}\right)+u_{N} \quad \text { for } u^{N} \in Z^{N}, u_{N} \in Z_{N}
$$

ProOF. Recall that $\Phi=e^{T J A}(I+\widetilde{\Phi})$. First, we observe that for $|t| \leq T$, any $R>0$ and $u, v \in B_{R}(Z)$ we have

$$
\left\|\widetilde{\Phi}(u)-\Pi^{N} \widetilde{\Phi}(u)\right\|_{Z} \leq \varepsilon_{1}(N) \underset{N \rightarrow+\infty}{\longrightarrow} 0 .
$$

Indeed, as $K=\bigcup_{|t| \leq T} \widetilde{\Phi}\left(B_{r}(Z)\right)$ is precompact in $Z$ (by (H3)), then (6) results from the following statement

$$
\sup _{u \in K}\left\|u-\Pi^{N} u\right\|_{N \rightarrow+\infty}^{\longrightarrow} 0 .
$$

Suppose that the convergence does not hold, then we can find a sequence $\left(u_{n}\right)$ in $K$ such that $\left\|\left(I-\Pi^{n}\right) u_{n}\right\| \geq \varepsilon>0$. As $K$ is precompact there exists a subsequence $\left(u_{n_{j}}\right)$ such that $u_{n_{j}} \rightarrow u$. For $n_{j}$ sufficiently large we have

$$
\left\|\left(I-\Pi^{n_{j}}\right)(u)\right\| \leq \varepsilon / 2, \quad\left\|u_{n_{j}}-u\right\| \leq \varepsilon / 2 .
$$

Hence $\left\|\left(I-\Pi^{n_{j}}\right)\left(u_{n_{j}}\right)\right\| \leq \varepsilon$ and we get a contradiction. 
Now we set $h_{N}=h \circ \Pi^{N}$. Then $\nabla h_{N}=\Pi^{N} \nabla h \Pi^{N}$. We define $\Phi^{N}$ the time $T$ flow of the equation

$$
\dot{v}=J\left(A v+\nabla h_{N}(v)\right)
$$

or, equivalently, $v=v^{N}+v_{N} \in Z^{N}+Z_{N}$ and

$$
\left\{\begin{array}{l}
\dot{v}^{N}=J\left(A v^{N}+\Pi^{N} \nabla h\left(v^{N}\right)\right) \\
\dot{v}_{N}=J A v_{N}
\end{array}\right.
$$

We write $\Phi^{N}=e^{T J A}\left(I+\widetilde{\Phi}_{N}\right)$.

Since $\widetilde{\Phi}_{N}=0$ outside $Z^{N}, \widetilde{\Phi}_{N}$ has the desired form (5). Define

$$
\widetilde{\Phi}_{\varepsilon}=\left(\widetilde{\Phi}-\widetilde{\Phi}_{N}\right)\left(I+\widetilde{\Phi}_{N}\right)^{-1},
$$

so we have

$$
e^{T J A}\left(I+\widetilde{\Phi}_{\varepsilon}\right)\left(I+\widetilde{\Phi}_{N}\right)=e^{T J A}(I+\widetilde{\Phi})=\Phi .
$$

Next we estimate the difference $\widetilde{\Phi}-\widetilde{\Phi}_{N}$. For $u \in B_{R}(Z)$ we have

$$
\begin{array}{r}
\left\|\widetilde{\Phi}(u)-\widetilde{\Phi}_{N}(u)\right\|_{Z} \leq\left\|\widetilde{\Phi}(u)-\Pi^{N} \widetilde{\Phi}(u)\right\|_{Z}+\left\|\Pi^{N} \widetilde{\Phi}(u)-\Pi^{N} \widetilde{\Phi}\left(\Pi^{N} u\right)\right\|_{Z} \\
+\left\|\Pi^{N} \widetilde{\Phi}\left(\Pi^{N} u\right)-\widetilde{\Phi}_{N}(u)\right\|_{Z} .
\end{array}
$$

Hence by (6) and assumption (H3), for $u \in B_{R}(Z)$ we have

$$
\left\|\widetilde{\Phi}(u)-\widetilde{\Phi}_{N}(u)\right\|_{Z} \leq C \varepsilon(N) \underset{N \rightarrow+\infty}{\longrightarrow} 0,
$$

so for $u \in\left(I+\widetilde{\Phi}_{N}\right)\left(B_{R}(Z)\right)$

$$
\left\|\widetilde{\Phi}_{\varepsilon}(u)\right\|_{Z} \leq \varepsilon(N) \underset{N \rightarrow+\infty}{\longrightarrow} 0 .
$$

2.3. Symplectic capacities and non-squeezing theorem.

2.3.1. Capacities in finite-dimentional space. Consider $\mathbb{R}^{2 n}$ supplied with the standard symplectic structure, that is $\omega(x, y)=\langle J x, y\rangle$ where

$$
J=\left(\begin{array}{cc}
0 & -I \\
I & 0
\end{array}\right) .
$$

For $f: \mathbb{R}^{2 n} \rightarrow \mathbb{R}$ a smooth function we define the hamiltonian vectorfield

$$
X_{f}=J \nabla f .
$$

Definition 2.4. Let $\mathcal{O}$ an open set of $\mathbb{R}^{2 n}, f \in C^{\infty}(\mathcal{O})$ and $m>0$. The function $f$ is called $m$-admissible if

- $0 \leq f(x) \leq m$ for $x \in \mathcal{O}$, and $f$ vanishes on a nonempty open set of $\mathcal{O}$, and $\left.f\right|_{\partial \mathcal{O}}=m$.

- The set $\{z / f(z)<m\}$ is bounded and the distance from this set to $\partial \mathcal{O}$ is $d(f)>0$.

Following [6] we define the capacity $c_{2 n}(\mathcal{O})$ of an open set $\mathcal{O}$ of $\mathbb{R}^{2 n}$ as $c_{2 n}(\mathcal{O})=\inf \left\{m_{*} /\right.$ for each $m>m_{*}$ and each $m$-admissible function $f$ in $\mathcal{O}$ the vectorfield $X_{f}$ has a non constant periodic solution of period $\left.\leq 1\right\}$. 
THEOREM 2.5. $c_{2 n}$ is a symplectic capacity, that is

- if $\mathcal{O}_{1} \subset \mathcal{O}_{2}$ then $c_{2 n}\left(\mathcal{O}_{1}\right) \leq c_{2 n}\left(\mathcal{O}_{2}\right)$ and if $\varphi: \mathcal{O} \rightarrow \mathbb{R}^{2 n}$ is a symplectic diffeomorphism then $c_{2 n}(\mathcal{O})=$ $c_{2 n}(\varphi(\mathcal{O}))$.

- $c_{2 n}(\lambda \mathcal{O})=\lambda^{2} c_{2 n}(\mathcal{O})$.

- $c_{2 n}\left(B_{1}\right)=c_{2 n}\left(\mathcal{C}_{r, 1}\right)=\pi$ where

$B_{r}=\left\{(p, q) / \sum\left(p_{j}^{2}+q_{j}^{2}\right)<r^{2}\right\}$, and $\mathcal{C}_{r, 1}=\left\{(p, q) /\left(p_{1}^{2}+q_{1}^{2}\right)<r^{2}\right\}$.

See $[6]$ for a proof. An immediate consequence of this theorem is the nonsqueezing theorem of M. Gromov [5].

THEOREM 2.6. The ball $B_{r}$ can be symplecticaly embedded into the cylinder $\mathcal{C}_{R, 1}$ if and only if $r \leq R$.

2.3.2. Construction of a capacity on Hilbert spaces. In this section we define a symplectic capacity on Hilbert spaces which is invariant with respect to the flow of the equation (2). We will follow the construction of S. Kuksin (see [7]).

For $\mathcal{O}$ an open set of $Z$ we denote $\mathcal{O}^{n}=\mathcal{O} \cap Z^{n}$ and observe that $\partial \mathcal{O}^{n} \subset$ $\partial \mathcal{O} \cap Z^{n}$

Definition 2.7. Let $f \in C^{\infty}(\mathcal{O})$ and $m>0$. The function $f$ is called $m$ admissible if

- $0 \leq f(x) \leq m$ for $x \in \mathcal{O}$, and $f$ vanishes on a nonempty open set of $\mathcal{O}$, and $\left.f\right|_{\partial \mathcal{O}}=m$.

- The set $\{z / f(z)<m\}$ is bounded and the distance from this set to $\partial \mathcal{O}$ is $d(f)>0$.

REMARK 2.8. If $f$ is $m$-admissible, denoting $\operatorname{supp}(f)=\{z / 0<f(z)<m\}$ we have

$$
\begin{aligned}
\operatorname{dist}\left(f^{-1}(0), \partial \mathcal{O}\right) & \geq d(f), \\
\operatorname{dist}(\operatorname{supp}(f), \partial \mathcal{O}) & \geq d(f) .
\end{aligned}
$$

Denote $f_{n}=\left.f\right|_{\mathcal{O}^{n}}$ and consider $X_{f_{n}}$ the corresponding hamiltonian vectorfield on $\mathcal{O}^{n}$.

Definition 2.9. A $T$-periodic trajectory of $X_{f_{n}}$ is called fast if it is not a stationnary point and $T \leq 1$.

A $m$-admissible function $f$ is called fast if there exists $n_{0}$ (depending on $f$ ) such that for all $n \geq n_{0}$ the vectorfield $X_{f_{n}}$ has a fast solution.

LEMMA 2.10. Each periodic trajectory of $X_{f_{n}}$ is contained in $\operatorname{supp}(f) \cap Z^{n}$.

Proof. Pick $z \in \mathcal{O}^{n} \backslash \operatorname{supp}(f), f_{n}$ takes either its minimal or maximal value in $z$, hence $X_{f_{n}}(z)=0$. Therefore $z$ is a stationnary point and a fast trajectory cannot pass through it.

We are now in position to define a capacity $c$.

Definition 2.11. For an open set $\mathcal{O}$ of $Z$ its capacity equals to $c(\mathcal{O})=\inf \left\{m_{*} /\right.$ each $m$-admissible function with $m>m_{*}$ is fast $\}$.

Proposition 2.12. Assume that $\mathcal{O}_{1}, \mathcal{O}_{2}$ and $\mathcal{O}$ are open sets of $Z$ and $\lambda \neq 0$

(1) if $\mathcal{O}_{1} \subset \mathcal{O}_{2}$ then $c\left(\mathcal{O}_{1}\right) \leq c\left(\mathcal{O}_{2}\right)$; 
(2) $c(\lambda \mathcal{O})=\lambda^{2} c(\mathcal{O})$.

Proof. (1) Assume $m<c\left(\mathcal{O}_{1}\right)$, by definition of $c$ there exists a $m$-admissible function $f$ of $\mathcal{O}_{1}$ which is not fast. Hence, there exists a sequence $\left(n_{j}\right) \rightarrow+\infty$ such that for every $j \in \mathbb{N}, X_{f_{n_{j}}}$ has no fast periodic trajectory. Define $\widetilde{f}$ on $\mathcal{O}_{2}$ by

$$
\widetilde{f}(x)= \begin{cases}f(x) & \text { if } x \in \mathcal{O}_{1} \\ m & \text { otherwise }\end{cases}
$$

The function $\widetilde{f}$ is clearly $m$-admissible on $\mathcal{O}_{2}$.

By lemma 2.10 , for each $j \in \mathbb{N}$, each fast solution $x(t)$ of $X_{\widetilde{f}_{n_{j}}}$ lies in $\operatorname{supp} \tilde{f} \cap$ $Z^{n_{j}}=\operatorname{supp} f \cap Z^{n_{j}}$. Hence $x(t)$ is a fast trajectory of $X_{f_{n_{j}}}\left(X_{\widetilde{f}_{n_{j}}}\right.$ and $X_{f_{n_{j}}}$ are the same vectorfields on $\operatorname{supp}(f)$ by definition of $\operatorname{supp}(f))$.

Therefore, for each $j \in \mathbb{N}$ the vectorfield $X_{\widetilde{f}_{n_{j}}}$ of $\mathcal{O}_{2}$ has no fast trajectory. Hence $\tilde{f}$ is $m$-admissible but is not fast. Thus $c\left(\mathcal{O}_{2}\right) \geq m$, and the first assertion follows.

(2) Define $f^{\lambda}=\lambda^{2} f\left(\lambda^{-1}\right.$ ) on $\lambda \mathcal{O}$. Clearly $f$ is $m$-admissible on $\mathcal{O}$ if and only if $f^{\lambda}$ is $\lambda^{2} m$-admissible on $\lambda \mathcal{O}$. Moreover $z(t) \in \mathcal{O}^{n}$ is a $T$-periodic trajectory of $X_{f_{n}}$ if and only if $\lambda z(t) \in \lambda \mathcal{O}^{n}$ is a $T$-periodic trajectory of $X_{f_{n}^{\lambda}}$. Therefore $c(\lambda \mathcal{O})=\lambda^{2} c(\mathcal{O})$.

LEMma 2.13. If $F: Z \rightarrow Z$ has the form

$$
F\left(z^{n}+z_{n}\right)=F^{n}\left(z^{n}\right)+z_{n} \quad z=z^{n}+z_{n} \in Z=Z^{n} \oplus Z_{n}
$$

with $F^{n}$ a symplectic diffeomorphism of $Z^{n}$, then $c(\mathcal{O})=c(F(\mathcal{O}))$, for each open set $\mathcal{O}$ of $Z$.

Proof. We observe that if $f$ is $m$-admissible in $F(\mathcal{O})$ and $f$ is fast then $f \circ F$ is $m$-admissible in $\mathcal{O}$ and $f \circ F$ is fast. Indeed $F^{*}: f \mapsto f \circ F$ clearly sends $m$-admissible functions in $F(\mathcal{O})$ to similar ones in $\mathcal{O}$, and for $p \geq n$ it tranforms $X_{(f \circ F)^{p}}$ into $X_{f^{p}}$. Hence admissible and fast functions are preserved by $F$ and its inverse ( $F$ is the identity outside of $Z^{n}$ which is a finite-dimentional space), and the result follows.

Proposition 2.14. For each open set $\mathcal{O}$ of $Z$ and $\xi$ in $Z$, we have

$$
c(\mathcal{O})=c(\mathcal{O}+\xi) .
$$

Proof. Denote $\mathcal{O}_{\xi}=\mathcal{O}+\xi$. It is sufficient to prove that $c(\mathcal{O}) \leq c(\mathcal{O}+\xi)$ (change $\xi$ into $-\xi$ ).

Denote $\xi=\xi^{n_{0}}+\xi_{n_{0}} \in Z^{n_{0}}+Z_{n_{0}}\left(n_{0}\right.$ will be fixed later) and $\mathcal{O}_{1}=\mathcal{O}+\xi^{n_{0}}$. By lemma $2.13 c\left(\mathcal{O}_{1}\right)=c(\mathcal{O})$. We also remark that $\mathcal{O}_{\xi}=\mathcal{O}_{1}+\xi_{n_{0}}$.

Take any $m$-admissible function $f$ on $\mathcal{O}_{\xi}$ with $m>c(\mathcal{O})$. We wish to check that $f$ is fast.

Since $\partial \mathcal{O}_{\xi} \subset \partial \mathcal{O}_{1}+\xi_{n_{0}}$ and $\left\|\xi_{n}\right\| \underset{n \rightarrow+\infty}{\longrightarrow} 0$, we have

$$
\operatorname{dist}\left(\partial \mathcal{O}_{1}, \partial \mathcal{O}_{\xi}\right) \leq \operatorname{dist}\left(\partial \mathcal{O}_{1}, \partial \mathcal{O}_{1}+\xi_{n_{0}}\right) \leq\left\|\xi_{n_{0}}\right\|_{n_{0} \rightarrow+\infty}^{\longrightarrow} 0 .
$$

Pick $n_{0}$ such that

$$
\operatorname{dist}\left(\partial \mathcal{O}_{1}, \partial \mathcal{O}_{\xi}\right) \leq\left\|\xi_{n_{0}}\right\|<\frac{1}{2} d(f)
$$

We extend $f$ outside $O_{\xi}$ with $f(z)=m$ if $z \notin \mathcal{O}_{\xi}$ and we denote $\tilde{f}$ its restriction to $\mathcal{O}_{1}$. 
$f$ equals $m$ on a $d(f)$-neighbourhood of $\partial \mathcal{O}_{\xi}$. By (8), we deduce that $\widetilde{f}$ equals $m$ on a $\frac{1}{2} d(f)$-neighbourhood of $\partial \mathcal{O}_{1}$.

By remark 2.8 we have $\operatorname{dist}\left(f^{-1}(0), \partial \mathcal{O}_{\xi}\right) \geq d(f)$. Hence, by (8), we have $\operatorname{dist}\left(f^{-1}(0), \partial \mathcal{O}_{1}\right) \geq \frac{1}{2} d(f)$, and in particular $\widetilde{f}$ vanishes on a nonempty open set of $\mathcal{O}_{1} \cap \mathcal{O}_{\xi} \subset \mathcal{O}_{1}$. Therefore $\widetilde{f}$ is $m$-admissible.

Since $c\left(\mathcal{O}_{1}\right)=c(\mathcal{O})<m$, it follows that $X_{\tilde{f}_{n}}$ has a fast trajectory in $\mathcal{O}_{1}^{n}$ if $n \geq n_{0}$ is sufficiently large. By lemma 2.10 this trajectory lies in $\operatorname{supp} \widetilde{f}=\operatorname{supp} f \subset$ $\mathcal{O}_{1} \cap \mathcal{O}$. Hence this trajectory is a fast solution of $X_{f_{n}}$, and the function $f$ is fast.

If $\boldsymbol{r}=\left(r_{j}\right)_{j \in \mathbb{N}^{*}}$ is a sequence of $\mathbb{R}_{+}^{*} \cup\{+\infty\}$ with $0<r=\inf _{j \in \mathbb{N}^{*}} r_{j}<+\infty$, we define

$$
\begin{gathered}
D(\boldsymbol{r})=\left\{z=\sum_{j=1}^{+\infty} p_{j} \varphi_{j}^{+}+q_{j} \varphi_{j}^{-} / \forall j \in \mathbb{N}, p_{j}^{2}+q_{j}^{2}<r_{j}^{2}\right\}, \\
E(\boldsymbol{r})=\left\{z=\sum_{j=1}^{+\infty} p_{j} \varphi_{j}^{+}+q_{j} \varphi_{j}^{-} / \sum_{j=1}^{+\infty} \frac{p_{j}^{2}+q_{j}^{2}}{r_{j}^{2}}<1\right\} .
\end{gathered}
$$

Remark that if $\boldsymbol{r}=(r,+\infty, \ldots,+\infty), D(\boldsymbol{r})$ is a symplectic cylinder $\mathcal{C}_{r, 1}$.

Theorem 2.15. We have $c(E(\boldsymbol{r}))=c(D(\boldsymbol{r}))=\pi r^{2}$

Proof. We have to check the following inequalities

(1) $c(E(\boldsymbol{r})) \geq \pi r^{2}$

(2) $c(D(\boldsymbol{r})) \leq \pi r^{2}$

then we will conclude by proposition 2.12 . 2.12).

(1) It is sufficient to prove that $c\left(B_{1}\right) \geq \pi$ (then the result follows by proposition

Define $m=\pi-\varepsilon$. Choose $f:[0,1] \rightarrow \mathbb{R}_{+}$satisfying :

$\left\{\begin{array}{l}0 \leq f^{\prime}(t)<\pi \text { for } t \in[0,1] \\ f(t)=0 \text { for } t \text { near } 0 \\ f(t)=m \text { for } t \text { near } 1\end{array}\right.$

Then, define $H(x)=f\left(\|x\|^{2}\right)$ for $x$ in $B(1) . H$ is $m$-admissible. We want to prove that $H$ is not fast. Consider

$$
H_{n}(x)=f\left(\sum_{j=1}^{n}\left(p_{j}^{2}+q_{j}^{2}\right)\right), \quad \text { where } x=\sum_{j}\left(p_{j} \varphi_{j}^{+}+q_{j} \varphi_{j}^{-}\right) .
$$

Using the variables $I_{j}=\frac{1}{2}\left(p_{j}^{2}+q_{j}^{2}\right)$ and $\theta_{j}=\arctan \left(\frac{p_{j}}{q_{j}}\right)$ we observe that nonconstant periodic solutions corresponding to this hamiltonian has a period $T>1$. Hence $X_{H_{n}}$ has no fast trajectory and $H$ is not fast.

(2) Denote $\mathcal{O}=D(\boldsymbol{r})$. Pick $m>\pi r^{2}$ and $f$ a $m$-admissible function in $\mathcal{O}$. Since $f^{-1}(0)$ is not empty, there exists $n$ such that $f^{-1}(0) \cap Z^{n} \neq \emptyset$. Denote $f_{n}=\left.f\right|_{\mathcal{O}^{n}}$. Since $\partial \mathcal{O}^{n} \subset \partial \mathcal{O}$, we deduce that $f_{n}$ equals $m$ on a neighbourhood of $\partial \mathcal{O}^{n}$. Hence $f_{n}$ is $m$-admissible.

Since $c_{2 n}\left(\mathcal{O}^{n}\right)=\pi \min _{1 \leq j \leq n} r_{j}^{2}$, we have

$$
c_{2 n}\left(\mathcal{O}^{n}\right) \underset{n \rightarrow+\infty}{\longrightarrow} \pi \inf _{j \geq 1} r_{j}^{2}=\pi r^{2}<m .
$$


Hence, for $n$ sufficiently large $c_{2 n}\left(\mathcal{O}^{n}\right)<m$. Therefore $X_{f_{n}}$ has a fast periodic trajectory and the function $f$ is fast.

Corollary 2.16. We have $c\left(B_{r}\right)=c\left(\mathcal{C}_{r, 1}\right)=\pi r^{2}$,

and for each bounded open set $\mathcal{O}$ of $Z$ we have $0<c(\mathcal{O})<+\infty$.

The essential property of the capacity $c$ is its invariance with respect to the flow maps of PDEs satisfying assumptions (H1), (H2) and (H3). In fact the nonsqueezing theorem 2.1 is a consequence of the following result.

THEOREM 2.17. Let $\Phi_{T}$ the flow of an equation (2) satisfying the assumptions (H1), (H2) and (H3). For any open set $\mathcal{O}$ of $Z$ we have

$$
c\left(\Phi_{T}(\mathcal{O})\right)=c(\mathcal{O}) .
$$

Proof. Let us denote $\Phi=\Phi_{T}$ and $\mathcal{Q}=\Phi(\mathcal{O})$. One easily checks that $\Phi^{-1}$ satisfies $(\mathrm{H} 1),(\mathrm{H} 2)$ and $(\mathrm{H} 3)$, therefore it is sufficient to prove that $c(\mathcal{Q}) \leq c(\mathcal{O})$.

Take any $m>c(\mathcal{O})$ and any $f m$-admissible in $\mathcal{Q}$. We want to prove that $f$ is fast.

Since $f$ is $m$-admissible there exists $R>0$ such that $\operatorname{supp} f \subset B_{R}$. Define $R_{1}=R+d(f), \mathcal{Q}^{\prime}=\mathcal{Q} \cap B_{R^{\prime}}$ and $\mathcal{O}^{\prime}=\Phi^{-1}\left(\mathcal{Q}^{\prime}\right)$. By assumption $\mathcal{O}^{\prime}$ is bounded, hence there exists $R^{\prime}$ such that $\mathcal{O}^{\prime} \subset B_{R^{\prime}}$. Moreover we clearly have $\mathcal{O}^{\prime} \subset \mathcal{O}$, thus by proposition 2.12

$$
c\left(\mathcal{O}^{\prime}\right) \leq c(\mathcal{O}) .
$$

We apply lemma 2.3 with $N$ so large that $\varepsilon<\frac{1}{2} d(f)$, and we use the notations of the lemma 2.3: $\Phi=e^{T J A}\left(I+\widetilde{\Phi}_{\varepsilon}\right)\left(I+\widetilde{\Phi}_{N}\right)$. We denote $\mathcal{O}_{1}$ and $\mathcal{O}_{2}$ the intermediate domains which arrise from the decomposition

$$
\mathcal{O}^{\prime} \stackrel{I+\widetilde{\Phi}_{N}}{\longrightarrow} \mathcal{O}_{1} \stackrel{I+\widetilde{\Phi}_{\varepsilon}}{\longrightarrow} \mathcal{O}_{2} \stackrel{e^{T J A}}{\longrightarrow} \mathcal{Q}^{\prime}
$$

We also denote

$$
f_{2}=\left.\left(f \circ e^{T J A}\right)\right|_{\mathcal{O}_{2}} .
$$

Observe that $f_{2}$ is $m$-admissible on $\mathcal{O}_{2}$. Indeed $f$ is $m$-admissible on $\mathcal{Q}$ and also on $\mathcal{Q}^{\prime}$ (by definition of $\mathcal{Q}^{\prime}$ ). Since $e^{t J A}$ is an isometry, $f_{2}$ is $m$-admissible.

Then, we extend $f_{2}$ as $m$ outside $\mathcal{O}_{2}$, and we denote $\widetilde{f}$ its restriction to $\mathcal{O}_{1}$. By (4) the $\varepsilon$-neighbourhood of $\partial \mathcal{O}_{1}$ is contained in the $2 \varepsilon$-neighbourhood of $\partial \mathcal{O}_{2}$. Since $\varepsilon<\frac{1}{2} d(f)$, we deduce that $\widetilde{f}$ equals $m$ on a neighbourhood of $\partial \mathcal{O}_{1}$. Moreover $\tilde{f}^{-1}(0)=f_{2}^{-1}(0) \subset \mathcal{O}_{1} \cap \mathcal{O}_{2}$. Indeed by remark 2.8

$$
\begin{aligned}
\operatorname{dist}\left(f_{2}^{-1}(0), \partial \mathcal{O}_{2}\right) & \geq d(f) \\
\text { and } \operatorname{dist}\left(\partial \mathcal{O}_{1}, \partial \mathcal{O}_{2}\right) & \leq \frac{1}{2} d(f) .
\end{aligned}
$$

Hence $\tilde{f}$ is $m$-admissible on $\mathcal{O}_{1}$.

Using lemma 2.13 and (9), we deduce that

$$
c\left(\mathcal{O}_{1}\right)=c\left(\left(I+\widetilde{\Phi}_{N}\right)\left(\mathcal{O}^{\prime}\right)\right)=c\left(\mathcal{O}^{\prime}\right) \leq c(\mathcal{O})<m .
$$

Hence $\tilde{f}$ is $m$-admissible on $\mathcal{O}_{1}$ and $c\left(\mathcal{O}_{1}\right)<m$, thus $\tilde{f}$ is fast. So for $n$ sufficiently large, the vectorfield $X_{\widetilde{f}_{n}}$ (where $\widetilde{f}_{n}=\left.\widetilde{f}\right|_{\mathcal{O}_{1}^{n}}$ ) has a fast solution. By lemma 2.10 this solution lies in $\operatorname{supp} \widetilde{f}$ and by remark $2.8 \operatorname{supp} \widetilde{f}=\operatorname{supp} f_{2}$, so this solution is 
also a fast solution of $X_{f_{2}^{n}}$ (where $f_{2}^{n}=\left.f_{2}\right|_{\mathcal{O}_{2}^{n}}$ ). Hence $f_{2}$ is fast too. Finally $f$ is also fast $\left(f_{2}=\left.\left(f \circ e^{T J A}\right)\right|_{\mathcal{O}_{2}}\right)$.

\section{Application to the BBM equation}

In this section we prove that the BBM equation

$$
\left\{\begin{array}{l}
u_{t}+u_{x}+u u_{x}-u_{x x t}=0, \quad x \in \mathbb{T} \\
u(0, x)=u_{0}(x)
\end{array}\right.
$$

is globally well-posed in $H^{s}(\mathbb{T})$ for $s \geq 0$ (we will follow the proof given in [2] for $x \in \mathbb{R}$ ) and has the non-squeezing property (theorem 1.1).

3.1. Bilinear estimates. We start by two helpful inequalities.

Let $\varphi(k)=\frac{k}{1+k^{2}}$ and $\varphi(D)$ the Fourier multiplier operator defined by $\widehat{\varphi(D) u}(k)=$ $\varphi(k) \widehat{u}(k)$.

Lemma 3.1. Let $u \in H^{r}(\mathbb{T})$ and $v \in H^{r^{\prime}}(\mathbb{T})$ with $0 \leq r \leq s, 0 \leq r^{\prime} \leq s$ and $0 \leq 2 s-r-r^{\prime}<1 / 4$. Then

$$
\|\varphi(D)(u v)\|_{H^{s}} \leq C_{r, r^{\prime}, s}\|u\|_{H^{r}}\|v\|_{H^{r^{\prime}}}
$$

Proof. We want to prove

$$
\left\|\langle k\rangle^{s} \frac{k}{1+k^{2}} \widehat{u v}(k)\right\|_{\ell_{k}^{2}} \leq C\|u\|_{H^{r}}\|v\|_{H^{r^{\prime}}} .
$$

By duality it is sufficient to prove

$$
\left\langle\langle k\rangle^{s} \frac{k}{1+k^{2}} \widehat{u v}, \widehat{w}\right\rangle_{\ell^{2}} \leq C\|u\|_{H^{r}}\|v\|_{H^{r^{\prime}}}\|w\|_{L^{2}}
$$

that is

$$
I=\sum_{k \in \mathbb{Z}} k\langle k\rangle^{s-2} \widehat{u v}(k) \overline{\widehat{w}}(k) \leq C\|u\|_{H^{r}}\|v\|_{H^{r^{\prime}}}\|w\|_{L^{2}} .
$$

Let $f(k)=\langle k\rangle^{r} \widehat{u}(k), g(k)=\langle k\rangle^{r^{\prime}} \widehat{v}(k)$ and $h(k)=k\langle k\rangle^{-2\left(1+r+r^{\prime}-2 s\right)} \overline{\widehat{w}}(k)$. Since

$$
\widehat{u v}(k)=\sum_{l \in \mathbb{Z}} \widehat{u}(l) \widehat{v}(k-l)
$$

we have

$$
I=\sum_{k \in \mathbb{Z}} \sum_{l \in \mathbb{Z}} \frac{\langle k\rangle^{-3 s+2 r+2 r^{\prime}}}{\langle l\rangle^{r}\langle k-l\rangle^{r^{\prime}}} f(l) g(k-l) h(k) .
$$

We have $-2 s+r+r^{\prime} \leq 0$ and $-s+r \leq 0$ and $-s+r^{\prime} \leq 0$ so

$-3 s+2 r+2 r^{\prime}=-2 s+r+r^{\prime}+\left(-s+r^{\prime}\right)+r \leq r$ and $-3 s+2 r+2 r^{\prime} \leq r^{\prime}$. 
Hence $\frac{\langle k\rangle^{-3 s+2 r+2 r^{\prime}}}{\langle l\rangle^{r}\langle k-l\rangle^{r^{\prime}}}$ is bounded for $k$ and $l$ in $\mathbb{Z}$. Then (by Cauchy-Schwarz inequality and Young's inequality)

$$
\begin{aligned}
I & \lesssim \sum_{k \in \mathbb{Z}} \sum_{l \in \mathbb{Z}} f(l) g(k-l) h(k) \\
& \lesssim\|f\|_{\ell^{2}}\|g * h(-\cdot)\|_{\ell^{2}} \\
& \lesssim\|f\|_{\ell^{2}}\|g\|_{\ell^{2}}\|h\|_{\ell^{1}} \\
& \lesssim\|u\|_{H^{r}}\|v\|_{H^{r^{\prime}}}\|w\|_{L^{2}}\left\|\frac{k}{\left(1+k^{2}\right)^{1+r+r^{\prime}-2 s}}\right\|_{\ell_{k}^{2}} .
\end{aligned}
$$

Since $2 s-r-r^{\prime}<1 / 4$ we have $1+r+r^{\prime}-2 s>3 / 4$. Hence

$$
\left\|\frac{k}{\left(1+k^{2}\right)^{1+r+r^{\prime}-2 s}}\right\|_{\ell_{k}^{2}}<+\infty
$$

In subsection 3.3 we will use this lemma in the particular case $r=r^{\prime}=s \geq 0$, that is

$$
\|\varphi(D)(u v)\|_{H^{s}} \leq C_{s}\|u\|_{H^{s}}\|v\|_{H^{s}}
$$

whereas in subsection 3.4 and 3.5 we will need the general case $0 \leq r, r^{\prime}<s$.

Lemma 3.2. Let $u \in H^{r}(\mathbb{T})$ and $v \in H^{s}(\mathbb{T})$ with $0 \leq s \leq r$ and $r>\frac{1}{2}$, then

$$
\|\varphi(D)(u v)\|_{H^{s+1}} \leq C\|u\|_{H^{r}}\|v\|_{H^{s}} .
$$

Proof. Since $r>\frac{1}{2}$ and $r \geq s \geq 0$, the elements of $H^{r}(\mathbb{T})$ are multipliers in $H^{s}(\mathbb{T})$, which is to say

Hence

$$
\|u v\|_{H^{s}} \lesssim\|u\|_{H^{r}}\|v\|_{H^{s}}
$$

$$
\begin{aligned}
\|\varphi(D)(u v)\|_{H^{s+1}} & =\left\|\frac{\langle k\rangle^{s+1} k}{\langle k\rangle^{2}} \widehat{u v}\right\|_{\ell_{k}^{2}} \\
& \leq\left\|\langle k\rangle^{s} \widehat{u v}\right\|_{\ell_{k}^{2}} \\
& =\|u v\|_{H^{s}} \\
& \lesssim\|u\|_{H^{r}}\|v\|_{H^{s}}
\end{aligned}
$$

3.2. Hamiltonian formalism for BBM equation. Recall that BBM equation reads

$$
u_{t}+u_{x}+u u_{x}-u_{t x x}=0 .
$$

Let us prove that BBM equation is a hamiltonian equation (2).

First BBM can be written

$$
u_{t}=-\partial_{x}\left(1-\partial_{x}^{2}\right)^{-1}\left(u+\frac{u^{2}}{2}\right) .
$$

Denote $Z=H_{0}^{1 / 2}(\mathbb{T})=\left\{u \in H^{1 / 2} / \int_{\mathbb{T}} u=0\right\}$ with the following norm

$$
\|u\|_{Z}=\sum_{k \in \mathbb{Z} \backslash\{0\}} \frac{1+k^{2}}{k}\left(a_{k}^{2}+b_{k}^{2}\right)
$$


where $a_{k}$ and $b_{k}$ are the (real) Fourier coefficients of $u$.

Consider the Hilbert basis of $\mathrm{Z}$ given by

$$
\varphi_{n}^{+}(x)=\sqrt{\frac{n}{\pi\left(n^{2}+1\right)}} \cos (n x), \quad \varphi_{n}^{-}(x)=\sqrt{\frac{n}{\pi\left(n^{2}+1\right)}} \sin (n x) .
$$

We have $Z_{+}=H_{0}^{1 / 2+\varepsilon}<H_{0}^{1 / 2}<H_{0}^{1 / 2-\varepsilon}=Z_{-}$, where $\varepsilon>0$ will be fixed later.

Define

$$
H(u)=\int_{\mathbb{T}}\left(\frac{u(x)^{2}}{2}+\frac{u(x)^{3}}{6}\right) d x,
$$

we have

Assume

$$
\nabla_{L^{2}} H(u)=u+\frac{u^{2}}{2} .
$$

$$
u(t)=\sum_{n} p_{n}(t) \varphi_{n}^{+}+q_{n}(t) \varphi_{n}^{-}
$$

and

$$
\nabla_{L^{2}} H(u)=\sum_{n} \alpha_{n} \varphi_{n}^{+}+\beta_{n} \varphi_{n}^{-} .
$$

Denoting $\widetilde{H}(p, q)=H\left(\sum_{n} p_{n}(t) \varphi_{n}^{+}+q_{n}(t) \varphi_{n}^{-}\right)$we deduce that

$$
\frac{\partial \widetilde{H}}{\partial p_{n}}=\left\langle\nabla_{L^{2}} H(u), \varphi_{n}^{+}\right\rangle_{L^{2}}=\alpha_{n}\left\|\varphi_{n}^{+}\right\|_{L^{2}}^{2}=\frac{n \alpha_{n}}{1+n^{2}}
$$

and

Hence

$$
\frac{\partial \widetilde{H}}{\partial q_{n}}=\frac{n \beta_{n}}{1+n^{2}}
$$

$$
\begin{aligned}
\dot{u}=\sum_{n} \dot{p}_{n} \varphi_{n}^{+}+\dot{q}_{n} \varphi_{n}^{-} & =\left(1-\partial_{x}^{2}\right)^{-1} \partial_{x}\left(-\nabla_{L^{2}} H(u)\right) \\
& =\sum_{n} \frac{-n \alpha_{n}}{1+n^{2}} \varphi_{n}^{-}+\frac{n \beta_{n}}{1+n^{2}} \varphi_{n}^{+}
\end{aligned}
$$

so

$$
\left\{\begin{array}{c}
\dot{p}_{n}=\frac{n \beta_{n}}{1+n^{2}}=\frac{\partial \widetilde{H}}{\partial q_{n}} \\
\dot{q}_{n}=\frac{-n \alpha_{n}}{1+n^{2}}=-\frac{\partial \widetilde{H}}{\partial p_{n}}
\end{array}\right.
$$

That is $\dot{u}=J \nabla_{Z} H(u)$.

\subsection{Verification of (H1).}

3.3.1. Local well-posedness. Recall that $\varphi(k)=\frac{k}{1+k^{2}}$, the equation (10) can be written in the form :

$$
\left\{\begin{array}{l}
i u_{t}=\varphi(D) u+\frac{1}{2} \varphi(D) u^{2} \\
u(0, x)=u_{0}(x)
\end{array}\right.
$$

Let $e^{-i t \varphi(D)}$ be the unitary group defining the associated free evolution. That is, $e^{-i t \varphi(D)} u_{0}$ solves the Cauchy problem

$$
\left\{\begin{array}{l}
i u_{t}=\varphi(D) u \\
u(0, x)=u_{0}(x)
\end{array}\right.
$$


Then, (11) may be rewritten as the integral equation

$$
u(t)=e^{-i t \varphi(D)} u_{0}-\frac{i}{2} \int_{0}^{t} e^{-i(t-\tau) \varphi(D)} \varphi(D)\left(u(\tau)^{2}\right) d \tau=\mathcal{A}(u)(t, \cdot) .
$$

Let $X_{T}^{s}=C^{0}\left([-T, T], H^{s}(\mathbb{T})\right)$. The $H^{s}$ norm is clearly preserved by the free evolution, thus

$$
\left\|e^{-i t \varphi(D)} u_{0}\right\|_{X_{T}^{s}}=\|u\|_{H^{s}} .
$$

THEOREM 3.3. Let $s \geq 0$. For any $u_{0} \in H^{s}(\mathbb{T})$, there exist a time $T$ (depending on $u_{0}$ ) and a unique solution $u \in X_{T}^{s}$ of (10). The maximal existence time $T_{s}$ has the property that

$$
T_{s} \geq \frac{1}{4 C_{s}\left\|u_{0}\right\|_{H^{s}}}
$$

with $C_{s}$ the constant from lemma 3.1 (in the special case $r=r^{\prime}=s$ ).

Moreover, for $R>0$, let $T$ denote a uniform existence time for (10) with $u_{0} \in B_{R}\left(H^{s}(\mathbb{T})\right)$, then the map $\Phi: u_{0} \mapsto u$ is real-analytic from $B_{R}\left(H^{s}(\mathbb{T})\right)$ to $X_{T}^{s}$.

Proof. Let $R=2\left\|u_{0}\right\|_{H^{s}}$. For any $u \in B_{R}\left(X_{T}^{s}\right)$, by (13) and lemma 3.1 (with $r=r^{\prime}=s$ ) we have

$$
\begin{aligned}
\|\mathcal{A}(u)\|_{X_{T}^{s}} & \leq\left\|e^{-i t \varphi(D)} u_{0}\right\|_{X_{T}^{s}}+\frac{1}{2}\left\|\int_{0}^{t} e^{-i(t-\tau) \varphi(D)} \varphi\left(u(\tau)^{2}\right) d \tau\right\|_{X_{T}^{s}} \\
& \leq\left\|u_{0}\right\|_{H^{s}}+\frac{C_{s} T}{2}\|u\|_{X_{T}^{s}}^{2} \\
& \leq\left\|u_{0}\right\|_{H^{s}}+\frac{C_{s} T}{2} R^{2} \\
& \leq R \quad \text { for } T=\frac{2}{C_{s} R}
\end{aligned}
$$

and for any $u, v \in B_{R}\left(X_{T}^{s}\right.$ ), by lemma 3.1 (with $r=r^{\prime}=s$ ) we have

$$
\|\mathcal{A}(u)-\mathcal{A}(v)\|_{X_{T}^{s}} \leq \frac{C_{s} T}{2}\|u-v\|_{X_{T}^{s}}\|u+v\|_{X_{T}^{s}} \leq C_{s} T R\|u-v\|_{X_{T}^{s}} .
$$

Hence, $\mathcal{A}$ is a contraction mapping of $B_{R}\left(X_{T}^{s}\right)$ for $T=\frac{1}{2 C_{s} R}=\frac{1}{4 C_{s}\left\|u_{0}\right\|_{H^{s}}}$. Thus $\mathcal{A}$ has a unique fixed point which is a solution of (10) on time interval $[-T, T]$.

Let us consider now the smoothness of $\Phi$. Let $\Lambda: H^{s}(\mathbb{T}) \times X_{T}^{s} \longrightarrow X_{T}^{s}$ be defined as

$$
\Lambda\left(u_{0}, v\right)(t)=v(t)-e^{-i t \varphi(D)} u_{0}-\frac{i}{2} \int_{0}^{t} e^{-i(t-\tau) \varphi(D)} \varphi(D)\left(v(\tau)^{2}\right) d \tau
$$

Due to lemme 3.1 (with $r=r^{\prime}=s$ ), $\Lambda$ is a smooth map from $H^{s}(\mathbb{T}) \times X_{T}^{s}$ to $X_{T}^{s}$. Let $u \in X_{T}^{s}$ be the solution of (10) with initial data $u_{0} \in H^{s}(\mathbb{T})$, which is to say $\Lambda\left(u_{0}, u\right)=0$. Thus, the Fréchet derivative of $\Lambda$ with respect to the second variable is the linear map :

$$
\Lambda^{\prime}\left(u_{0}, u\right)(t)[h]=h-\int_{0}^{t} e^{-i(t-\tau) \varphi(D)} \varphi(D)(u(\tau) h(\tau)) d \tau
$$

Still by lemma 3.1 we get

$$
\left\|\int_{0}^{t} e^{-i(t-\tau) \varphi(D)} \varphi(D)(u(\tau) h(\tau)) d \tau\right\|_{X_{T}^{s}} \leq C T\|u\|_{H^{s}}\|h\|_{H^{s}} .
$$


So, for $T^{\prime}$ sufficiently small (depending only on $\left.\|u\|_{H^{s}}\right), \Lambda^{\prime}\left(u_{0}, u\right)(t)$ is invertible since it is of the form $I d+K$ with

$$
\|K\|_{\mathcal{B}\left(X_{T^{\prime}}^{s}, X_{T^{\prime}}^{s}\right)}<1
$$

where $\mathcal{B}\left(X_{T^{\prime}}^{s}, X_{T^{\prime}}^{s}\right)$ is the Banach space of bounded linear operators on $X_{T^{\prime}}^{s}$. Thus $\Phi: B_{R}\left(H^{s}(\mathbb{T})\right) \rightarrow X_{T}^{s}$ is real-analytic by Implicit Function Theorem.

\subsubsection{Global well-posedness.}

Theorem 3.4. The solution defined in theorem 3.3 is global in time.

Proof. Fix $T>0$. The aim is to show that corresponding to any initial data $u_{0} \in H^{s}$, there is a unique solution of (10) that lies in $X_{T}^{s}$. Because of theorem 3.3, this result is clear for data that is small enough in $H^{s}$, and it is sufficient to prove the existence of a solution corresponding to initial data of arbitrary size (uniqueness is a local issue). Fix $u_{0} \in H^{s}$ and let $N$ be such that

$$
\sum_{|k| \geq N}\langle k\rangle^{2 s}\left|\widehat{u_{0}}(k)\right|^{2} \leq T^{-2} .
$$

Such values of $N$ exist since $\langle k\rangle^{s}\left|\widehat{u_{0}}(k)\right|$ is in $\ell^{2}$. Define

$$
v_{0}(x)=\sum_{|k| \geq N} e^{i x k} \widehat{u_{0}}(k) .
$$

By theorem 3.3, there exists a unique $v \in X_{T}^{s}$ solution of (10) with initial data $v_{0}$. Split the initial data $u_{0}$ into two pieces: $u_{0}=v_{0}+w_{0}$; and consider the following Cauchy problem (where $v$ is now fixed)

$$
\left\{\begin{array}{l}
w_{t}-w_{x x t}+w_{x}+w w_{x}+(v w)_{x} \\
w(0, x)=w_{0}(x)
\end{array}\right.
$$

If there exists a solution $w$ of (14) in $X_{T}^{s}$ then $v+w$ will be a solution of (10) in $X_{T}^{s}$.

First, $w_{0}$ is in $H^{r}(\mathbb{T})$ for all $r>0$, in particular $w_{0} \in H^{1}(\mathbb{T})$. And (14) may be rewritten as the integral equation

$$
w(t, x)=e^{-i t \varphi(D)} w_{0}-\frac{i}{2} \int_{0}^{t} e^{-i(t-\tau) \varphi(D)} \varphi(D)\left(v w+w^{2}\right) d \tau=\mathcal{K}(w) .
$$

This problem can be solved locally in time on $H^{1}(\mathbb{T})$ by the same arguments used to prove theorem 3.3. Indeed for any $w \in B_{R}\left(X_{S}^{1}\right)$, by lemma 3.2 (with $r=1$ and $s=0$ ) and lemma 3.1 (with $r=r^{\prime}=s=1$ )

$$
\begin{aligned}
\|\mathcal{K}(w)\|_{X_{S}^{1}} & \leq\left\|w_{0}\right\|_{H^{1}}+C S\left(\|v\|_{X_{S}^{0}}\|w\|_{X_{S}^{1}}+\|w\|_{X_{S}^{1}}^{2}\right) \\
& \leq C S\|v\|_{X_{S}^{0}} R
\end{aligned}
$$

and for any $w_{1}$ and $w_{2}$ in $B_{R}\left(X_{S}^{1}\right)$

$$
\begin{aligned}
\| \mathcal{K}\left(w_{1}\right) & -\mathcal{K}\left(w_{2}\right) \|_{X_{S}^{1}} \\
& \leq C S\left(\|v\|_{X_{S}^{0}}\left\|w_{1}-w_{2}\right\|_{X_{S}^{1}}+\left\|w_{1}-w_{2}\right\|_{X_{S}^{1}}\left\|w_{1}+w_{2}\right\|_{X_{S}^{1}}\right) \\
& \leq C S\left(\|v\|_{X_{S}^{0}}+2 R\right)\left\|w_{1}-w_{2}\right\|_{X_{S}^{1}} .
\end{aligned}
$$

Hence, by (15) and (16), $\mathcal{K}$ has a unique fixed point in $X_{S}^{1}$. Therefore we have a solution $w$ in $X_{S}^{1}$ for a small time $S$. 
If we have an a priori bound on the $H^{1}$-norm of $w$ showing it was bounded on the interval $[-T, T]$ it would follow that a solution on $[-T, T]$ could be obtained.

The formal steps of this inequality are as follows (the justification is made by regularizing). Multiply the equation (14) by $w$, integrate over $\mathbb{T}$, and after integration by parts we get

$$
\frac{1}{2} \frac{d}{d t} \int_{\mathbb{T}}\left(w(t, x)^{2}+w_{x}(t, x)^{2}\right) d x-\int_{\mathbb{T}} v(t, x) w(t, x) w_{x}(t, x) d x=0 .
$$

By Hölder and Sobolev inequalities we deduce

$$
\begin{aligned}
\left|\int_{\mathbb{T}} v(t, x) w(t, x) w_{x}(t, x) d x\right| & \leq\|v(t, \cdot)\|_{L^{2}}\|w(t, \cdot)\|_{L^{\infty}}\left\|w_{x}(t, \cdot)\right\|_{L^{2}} \\
& \leq C\|v(t, \cdot)\|_{L^{2}}\|w(t, \cdot)\|_{H^{1}}^{2} .
\end{aligned}
$$

Hence

and by Gronwall's inequality

$$
\frac{d}{d t}\|w(t, \cdot)\|_{H^{1}}^{2} \leq 2 C\|v(t, \cdot)\|_{L^{2}}\|w(t, \cdot)\|_{H^{1}}^{2}
$$

$$
\|w(t, \cdot)\|_{H^{1}} \leq\left\|w_{0}\right\|_{H^{1}} \exp \left(C \int_{0}^{t}\|v(\tau, \cdot)\|_{L^{2}} d \tau\right) .
$$

We deduce from this a priori bound that the solution $w$ of (14) exists on the interval $[-T, T]$, and $v+w$ is a solution of $(10)$ in $X_{T}^{s}$.

\subsection{Verification of (H2).}

Proposition 3.5. For any $T>0, R>0$, and $s>0$ there exists $R^{\prime}$ such that

$$
\forall 0 \leq t \leq T, \Phi_{t}\left(B_{R}\left(H^{s}\right)\right) \subset B_{R^{\prime}}\left(H^{s}\right) .
$$

With $s=\frac{1}{2}$ we deduce that $\Phi$ satisfies (H2).

Proof. The result is clear for $s \geq 1$, so we assume that $0<s<1$. Fix $T>0$, $R>0$ and $u_{0}$ in $H^{s}$ such that $\left\|u_{0}\right\|_{H^{s}} \leq R$. Using the same idea as in theorem 3.4 split $u_{0}$ into two pieces $u_{0}=v_{0}+w_{0}$, where

$$
v_{0}=\sum_{|k| \geq N} \widehat{u_{0}}(k) e^{i k x}
$$

Using the same notations, let $v$ be the solution of BBM equation with the initial data $v_{0}$ and $w$ the solution of (14). We want to control $v$ and $w$ in $H^{s}$-norm.

Fix $\varepsilon>0$ such that $\varepsilon<1 / 8$ and $s-\varepsilon>0$, we have

$$
\left\|v_{0}\right\|_{H^{s-\varepsilon}} \leq N^{-\varepsilon}\left\|v_{0}\right\|_{H^{s}} \text {. }
$$

We choose $N=\left(\frac{4 R C}{T}\right)^{1 / \varepsilon}$ where $C$ is the constant of lemma 3.1. Hence we have

$$
\left\|v_{0}\right\|_{H^{s-\varepsilon}} \leq \frac{1}{4 C T}=M .
$$

By local theory (theorem 3.3) the flow map

$$
\Phi: B_{M}\left(H^{s-\varepsilon}\right) \longrightarrow X_{T}^{s-\varepsilon}
$$

is continuous. Since $H^{s} \cap B_{M}\left(H^{s-\varepsilon}\right)$ is precompact in $B_{M}\left(H^{s-\varepsilon}\right)$ we have

$$
\sup _{v_{0} \in H^{s} \cap B_{M}\left(H^{s-\varepsilon}\right)}\left\|\Phi\left(v_{0}\right)\right\|_{X^{s-\varepsilon}}=C_{1}(R, T) .
$$


By lemma 3.1 with $r=r^{\prime}=s-\varepsilon$ we have

$$
\|v\|_{X^{s}} \leq\left\|v_{0}\right\|_{H^{s}}+C T\|v\|_{X^{s-\varepsilon}}^{2} \leq R+C T C_{1}(R, T)^{2}=C_{2}(R, T) .
$$

The a priori bound on $w$ gives

$$
\begin{aligned}
\|w(t)\|_{H^{s}} \leq\|w(t)\|_{H^{1}} & \leq\left\|w_{0}\right\|_{H^{1}} \exp \left(C \int_{0}^{t}\|v(\tau, \cdot)\|_{L^{2}} d \tau\right) \\
& \leq N^{1-s}\left\|w_{0}\right\|_{H^{s}} e^{C T C_{2}(R, T)} \\
& \leq C_{3}(R, T) .
\end{aligned}
$$

Hence, we have

$$
\|u\|_{X_{T}^{s}} \leq C_{2}(R, T)+C_{3}(R, T)
$$

Corollary 3.6. For each $T>0$ and $s>0$, the flow map $\Phi: H^{s} \rightarrow X_{T}^{s}$ is real analytic.

Proof. Let $u_{0} \in H^{s}, R=\left\|u_{0}\right\|_{H^{s}}$ and $T>0$. By proposition 3.5, there exists $R^{\prime}$ such that $\Phi_{t}\left(B_{2 R}\left(H^{s}\right)\right) \subset B_{R^{\prime}}\left(H^{s}\right)$, for all $t \in[0, T]$. And by local theory (theorem 3.3) there exists a small time $\tau$ such that $\Phi: B_{R^{\prime}}\left(H^{s}\right) \rightarrow X_{\tau}^{s}$ is real analytic. Splitting the time intervalle $[0, T]$ into $\bigcup[k \tau,(k+1) \tau]$, we deduce that $\Phi: H^{s} \rightarrow X_{T}^{s}$ is real analytic.

3.5. Verification of (H3). Recalll that $\widetilde{\Phi}$ denote the non-linear part of the flow, that is $\Phi_{t}=e^{-i t \varphi(D)}\left(I+\widetilde{\Phi}_{t}\right)$. The assumption (H3) results from

Proposition 3.7. For any $u_{0}, v_{0} \in B_{R}\left(H^{1 / 2}(\mathbb{T})\right)$ we have the following estimate

$$
\left\|\widetilde{\Phi}\left(u_{0}\right)-\widetilde{\Phi}\left(v_{0}\right)\right\|_{X_{T}^{1 / 2+\varepsilon}} \leq C_{R, T, \varepsilon}\left\|u_{0}-v_{0}\right\|_{H^{1 / 2-\varepsilon}}
$$

for $0<\varepsilon<1 / 12$.

Proof. Let $0<\varepsilon<\frac{1}{12}, u_{0}$ and $v_{0}$ in $B_{R}\left(H^{1 / 2}\right)$. Denoting $u$ and $v$ the solutions of BBM equation with initial data $u_{0}$ and $v_{0}$. By lemma 3.1 with $s=\frac{1}{2}+\varepsilon$ and $r=\frac{1}{2}$ and $r^{\prime}=\frac{1}{2}-\varepsilon$ and $(\mathrm{H} 2)$ we have

$$
\begin{aligned}
\left\|\widetilde{\Phi}_{t}\left(u_{0}\right)-\widetilde{\Phi}_{t}\left(v_{0}\right)\right\|_{X_{T}^{1 / 2+\varepsilon}} & \leq C T\|u+v\|_{X_{T}^{1 / 2}}\|u-v\|_{X_{T}^{1 / 2-\varepsilon}} \\
& \leq 2 C T R_{R, T}^{\prime}\|u-v\|_{X_{T}^{1 / 2-\varepsilon}}
\end{aligned}
$$

Since $u_{0}$ and $v_{0}$ are in $B_{R}\left(H^{1 / 2}\right)$ and $\Phi$ is $C^{1}$ on $B_{R}\left(H^{1 / 2}\right)$ which is a relatively compact subset of $H^{1 / 2-\varepsilon}$ we have

$$
\begin{aligned}
& \|u-v\|_{X_{T}^{1 / 2-\varepsilon}}=\left\|\Phi_{t}\left(u_{0}\right)-\Phi_{t}\left(v_{0}\right)\right\|_{X_{T}^{1 / 2-\varepsilon}} \\
& \quad \leq \sup _{w_{0} \in B_{R}\left(H^{1 / 2}\right) \cap H^{1 / 2-\varepsilon}}\left(\left\|d \Phi\left(w_{0}\right)\right\|_{\mathcal{B}\left(H^{1 / 2-\varepsilon}, X_{T}^{1 / 2-\varepsilon}\right)}\right)\left\|u_{0}-v_{0}\right\|_{H^{1 / 2-\varepsilon}} \\
& \quad \leq C_{R, T, \varepsilon}\left\|u_{0}-v_{0}\right\|_{H^{1 / 2-\varepsilon}} .
\end{aligned}
$$


Hence, we can apply the non-squeezing theorem (theorem 2.1) and that proves the theorem 1.1.

Acknowledgments: I'm grateful to Nikolay Tzvetkov for introducing me to this subject and for his advices on my work. I would also like to thank Patrick Gérard for many helpful discussions.

I thank the referee for pointing out an error in a previous version of this paper.

\section{References}

[1] Thomas B. Benjamin, Jerry L. Bona, John J. Mahony, Model equations for long waves in nonlinear dispersive systems, Philosophical Transactions of the Royal Society of London 272, no. 1220 (1972), 47-78.

[2] Jerry L. Bona, Nikolay Tzvetkov, Sharp well-posedness results for the BBM equation, Discrete and Continuous Dynamical Systems 23, no. 4 (2009), 1241-1252.

[3] Jean Bourgain, Aspects of long time behaviour of solutions of nonlinear Hamiltonian evolution equations, Geometric and Functional Analysis 5, no. 2 (1995), 105-140.

[4] James Colliander, Markus Keel, Gigliola Staffilani, Hideo Takaoka, Terence Tao, Symplectic nonsqueezing of the Korteweg-de Vries flow, Acta Mathematica 195, no. 2 (2005), 197-252.

[5] Mikhail Gromov, Pseudo-holomorphic curves in symplectic manifolds, Inventiones Mathematicae 82, no. 2 (1985), 307-347.

[6] Helmut Hofer, Eduard Zehnder, Symplectic Invariants and Hamiltonian Dynamics, Birkhäuser, 1994.

[7] Sergeï Kuksin, Infinite-dimensional symplectic capacities and a squeezing theorem for Hamiltonian PDE's, Communications in Mathematical Physics 167 (1995), 531-552.

[8] Mahendra Panthee, On the ill-posedness result for the BBM equation, arXiv:1003.6098v1, preprint 2010.

University of Cergy-Pontoise, Department of Mathematics, CNRS, UmR 8088, F95000 CERgy-Pontoise

E-mail address: david.roumegoux@u-cergy.fr 